\title{
Kalman Filtering Techniques For Fault Detection And Diagnosis In Continuously Stirred Tank Reactor (Cstr)
}

\author{
P V Sunil Nag, Sabarish R P Nair,Gowtham M, Sibichakravarthy V, T D Shivendran, Manjunath S \\ Amrita School of Engineering/Dept. of Electronics and Communication Engineering, \\ Amrita VishwaVidyapeetham, Coimbatore, Tamil Nadu, India.
}

\begin{abstract}
Fault detection and isolation is one of the major challenges every chemical industry is concerned with, because it deals with the performance of the machinery. Fault detection methods play an essential role in reducing costs, increasing safety and minimizing the effect on the surroundings[1]. To study and compare various Fault detection and isolation techniques, the mathematical model of a continuously stirred tank reactor (CSTR) is implemented in MATLAB. The output, which is obtained from this model, is fed to Kalman filter, which in turn gives a residue that is used to detect the faults present in the system. The difference between the measured process variables and their estimate is called a residue[2]. The same method is used for detecting faults for non-linear state equations with Extended Kalman filter and the residue is obtained from the same. From the residues obtained from Kalman and Extended Kalman filter a conclusion is made that the Extended Kalman filter gives more accurate results.
\end{abstract}

\section{Introduction}

Fault detection and diagnosis is one of the important problems in the industries. The occurrence of even small fault may lead to huge losses[3].There are three main categories for the detection of faults:signal based methods,statistical methods and model based methods. We have chosen dual tank CSTR [3] for studying model based fault detection and diagnosis methods.

The innovation part of the Kalman filter acts as the residual. The Kalman filter is replaced by extended Kalman filter for the same system. In Kalman filter, only linear model can be used but in extended Kalman filter non-linear model can be directly used. By analyzing these residuals, we could detect the occurrence of the fault and the comparative study of Kalman filter and extended Kalman filter is made. Model based fault detection methods works on the principle of several measured variables to extract data on the possible changes caused by the faults [4]. These methods are mostly in the form of the process model equations. The model based FDI techniques are classified into: Observer-based methods, Parity space methods and Parameter identification based.

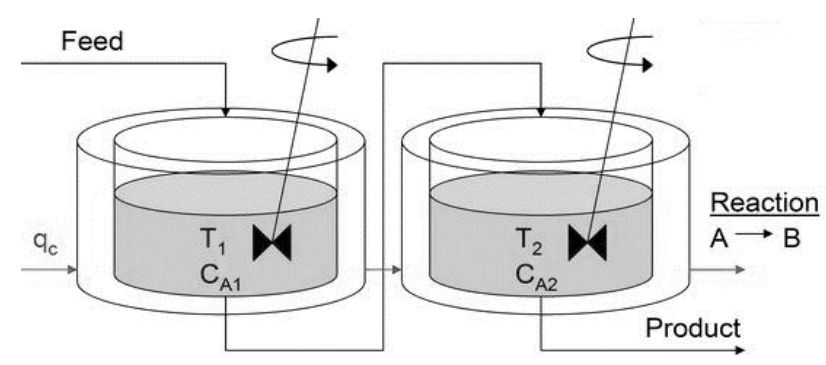

Fig. 1 The schematic of a two tank CSTR.

\section{Implementation and Results}

The input is given to the actual system as well as the Kalman filter. The output of both the actual system and the Kalman filter is compared and a residual is obtained which is used to identify the fault. The MATLAB simulation of two tank CSTR is developed. The output from this model is fed to Kalman filter. Kalman filter thus generates the residual which is observed to find faults. The Kalman filter is replaced with Extended Kalman filter for the same system and the residual obtained from Extended Kalman Filter is observed to find the faults.

The system we have selected to detect its fault has the following state space relations [3]:

$$
\begin{gathered}
C_{A 1}=\frac{q}{V_{1}}\left(C_{A f}-C_{A 1}\right)-e^{\left(-\frac{E}{R T_{1}}\right)} \\
\begin{aligned}
T_{1}=\frac{q}{V_{1}}\left(T_{f}-T_{1}\right) & +\frac{(-\Delta H) * k o * C_{A 1}}{\rho c p} * e^{\left(-\frac{E}{R T_{1}}\right)} \\
& +\frac{\rho_{c} c_{p c} q_{c}}{\rho c_{p} V_{1}}\left(1-e^{\frac{-h A_{1}}{q_{c} \rho_{c} c_{p c}}}\right)\left(T_{c f}-T_{1}\right)
\end{aligned} \\
C_{A 2}=\frac{q}{V_{2}}\left(C_{A 1}-C_{A 2}\right)-k o * C_{A 2} * e^{\left(-\frac{E}{R T_{2}}\right)} \\
T_{2}=\frac{q}{V_{2}}\left(T_{1}-T_{2}\right)+\frac{\operatorname{deltaH} * k o * C_{A 2}}{\rho c p} * e^{\left(-\frac{E}{R T_{2}}\right)}+\frac{\rho_{c} C_{p c} q_{c}}{\rho c_{p} V_{2}} \\
* q_{c}\left[1-e^{\frac{-h A_{2}}{q_{c} \rho_{c} C_{p c}}}\right] *\left[T_{1}-T_{2}\right. \\
\left.+e^{\frac{-h A_{1}}{q_{c} \rho_{c} c_{p c}}}\left(T_{c f}-T_{1}\right)\right]
\end{gathered}
$$

The standard state-space form is: $\mathrm{x}=\left[\mathrm{C}_{\mathrm{A} 1}, \mathrm{~T}_{1}, \mathrm{C}_{\mathrm{A} 2}, \mathrm{~T}_{2}\right], \mathrm{d}=\left[\mathrm{C}_{\mathrm{Af}}\right.$, $\left.\mathrm{T}_{\mathrm{cf}}\right], \mathrm{u}=\mathrm{q}_{\mathrm{c}}, \mathrm{y}=\mathrm{C}_{\mathrm{A} 2}$, here the first and second variables are the effluent concentration and temperature in the first tank and the third and fourth variables are the effluent concentration and temperature in the second tank. 
Inorder to implement the Extended Kalman Filter, the Jacobian matrix containing the partial derivatives of all the four states are found out.

The Jacobian is found by finding partial derivatives and writing it in the form [6]:

$\left[\begin{array}{llll}\frac{\partial f_{1}}{\partial_{x 1}} & \frac{\partial f_{1}}{\partial_{x 2}} & \frac{\partial f_{1}}{\partial_{x 3}} & \frac{\partial f_{1}}{\partial x 4} \\ \frac{\partial f_{2}}{\partial_{x 1}} & \frac{\partial f_{2}}{\partial_{x 2}} & \frac{\partial f_{2}}{\partial f_{3}} & \frac{\partial f_{2}}{\partial_{x 4}} \\ \frac{\partial f_{3}}{\partial_{x 1}} & \frac{\partial f_{3}}{\partial_{x 2}} & \frac{\partial f_{3}}{\partial x 3} & \frac{\partial f_{3}}{\partial_{x 4}} \\ \frac{\partial f_{4}}{\partial_{x 1}} & \frac{\partial f_{4}}{\partial_{x 2}} & \frac{\partial f_{4}}{\partial_{x 3}} & \frac{\partial f_{4}}{\partial_{x 4}}\end{array}\right]$

\section{Dual tank CSTR}

The dual tank CSTR system involves only four states $\mathrm{C}_{\mathrm{A} 1}$ $\mathrm{T}_{1} \mathrm{C}_{\mathrm{A} 2} \mathrm{~T}_{2}$. Therefore the matrices will be a $4 \times 4$ matrix and the input to the system is the coolant flow rate $\mathrm{q}_{\mathrm{c}}$ which is fixed at $120 \mathrm{~L} / \mathrm{min}$.

\section{Simulation of the system}

The system is simulated for 100 minutes without any faults.

\section{Dual Tank CSTR concentration and temperature}
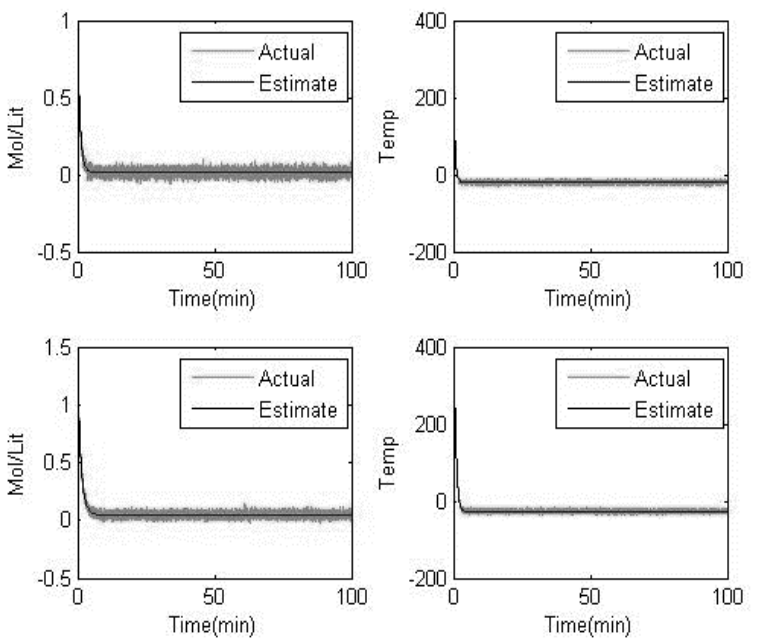

Fig. 2 System output showing the simulation for $100 \mathrm{~min}$ without any fault in dual tank CSTR (Temperature is in kelvin)
Dual Tank CSTR concentration and temperature residue
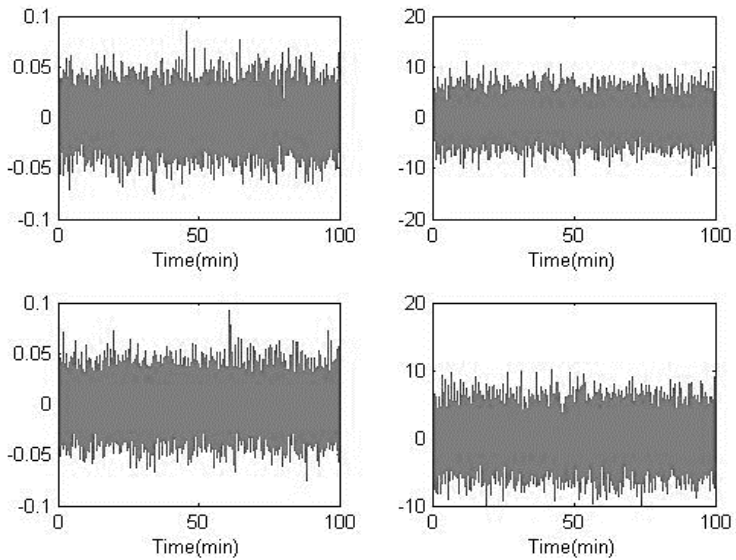

Fig. 3 Residue of all the four states in a dual tank CSTR. (Temperature is in kelvin)

\section{Introducing faults}

Faults are introduced from $20 \mathrm{~min}$ to $40 \mathrm{~min}$ in the actual system.

$C_{A 2}$ sensor fault $\left(C_{A 2}\right.$ - Concentration of tank 2):

\section{Concentration $\mathrm{C}_{\mathrm{A} 2}$ bias fault}
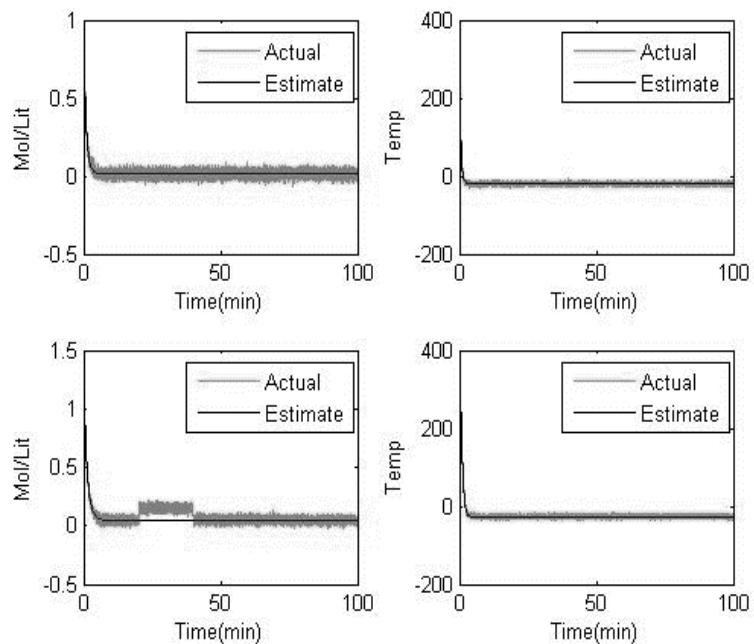

Fig. 4 Introduction of sensor fault $\left(\mathrm{C}_{\mathrm{A} 2}\right)$ in the system. (Temperature is in kelvin)

If there is a bias fault in the sensor of the $\mathrm{C}_{\mathrm{A} 2}$ then the residue shows a shift caused due to the bias in the sensor. 
Concentration $\mathrm{C}_{\mathrm{A} 2}$ bias fault residue
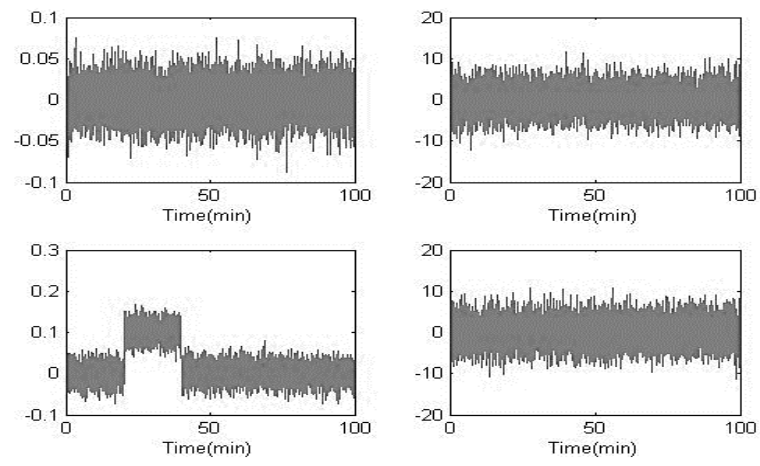

Fig. 5 Residue showing a shift caused due to bias in the sensor (Temperature is in kelvin)

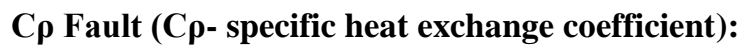

\section{Co parameter fault}
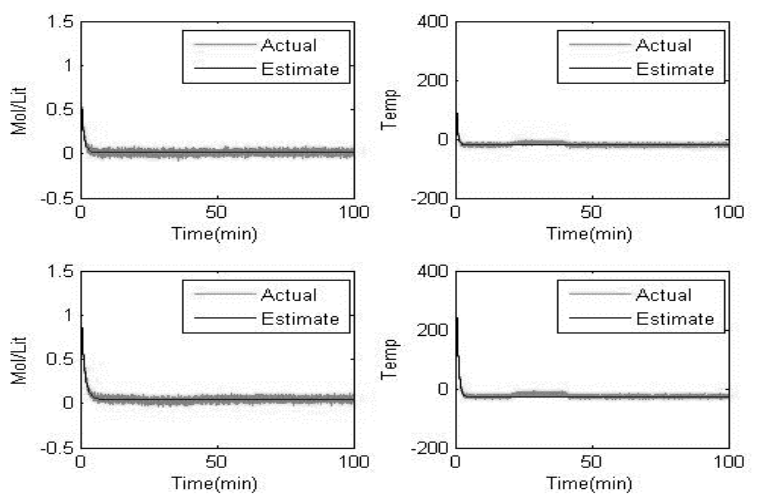

Fig. 6 Introduction of $\mathrm{C} \rho$ parameter change in the system (Temperature is in kelvin)

Introducing the $\mathrm{Cp}$ parameter change in the system the change is seen in the both the temperature and the concentration of tank 2 of the system. This can be clearly seen by observing the residue of the Kalman filter.

\section{C $\rho$ parameter fault residue $q$ parameter fault residue}
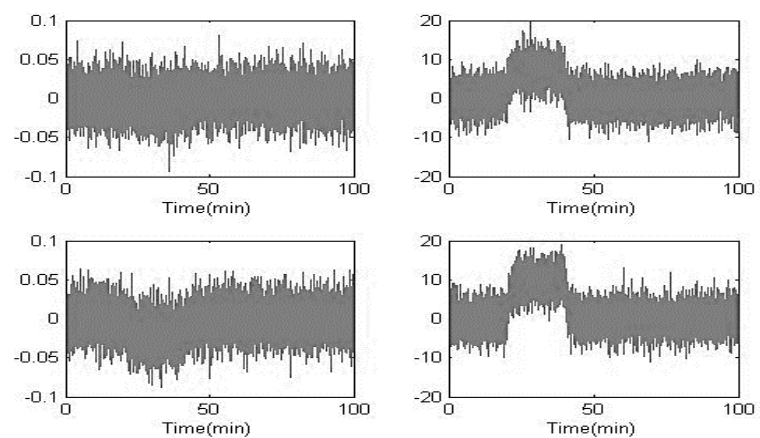

Fig. 7 Residue showing a shift caused due to $\mathrm{C} \rho$ parameter change in the system (Temperature is in kelvin)

\section{$q$ fault ( $q$ - feed flow rate): $q$ parameter fault}
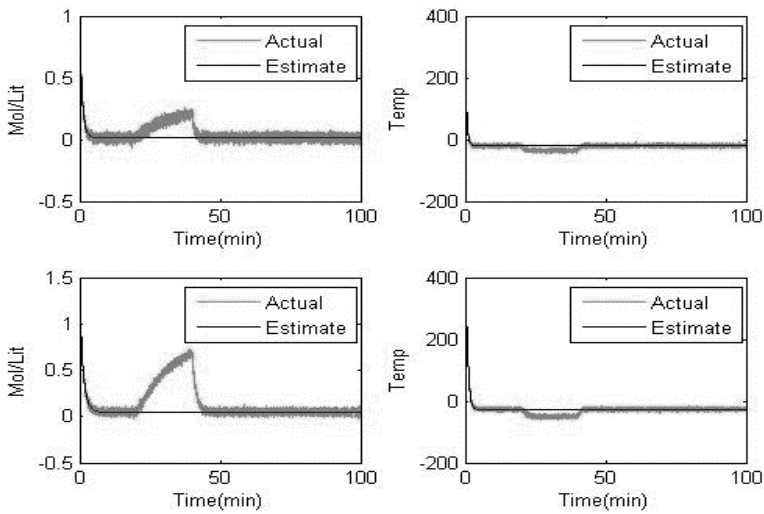

Fig. 8 Introduction of $q$ fault to the system (Temperature is in kelvin)

Introducing the $\mathrm{q}$ (input feed flow rate) parameter change in the system the change is seen both in the concentration and the temperature of both the tanks of the system. This can be clearly seen by observing the residue of the Kalman filter.
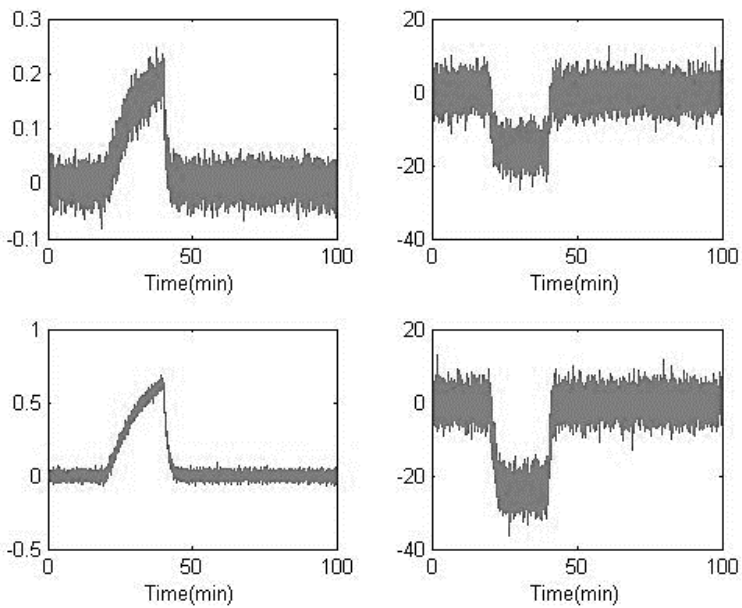

Fig. 9 Residue showing a shift caused due to $q$ fault (Temperature is in kelvin)

\section{Results of Extended Kalman Filter with Dual Tank CSTR}

Without Fault:

When there is no fault introduced in the system, the output of the system looks like the following graphs: 


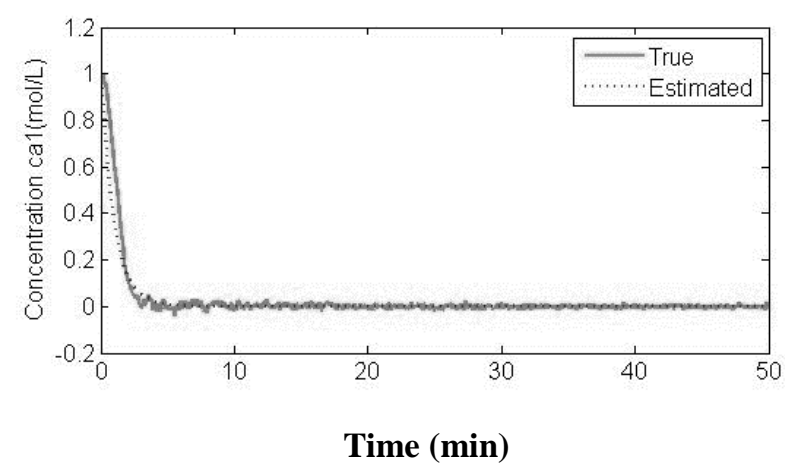

Fig.10 Graph showing the plot of concentration of tank 1 against time (duration 50 min)

True state is the output of the actual system with noise and the estimated shows the output of the EKF which is tracking the actual system output which is more accurate by removing the noise in the system.

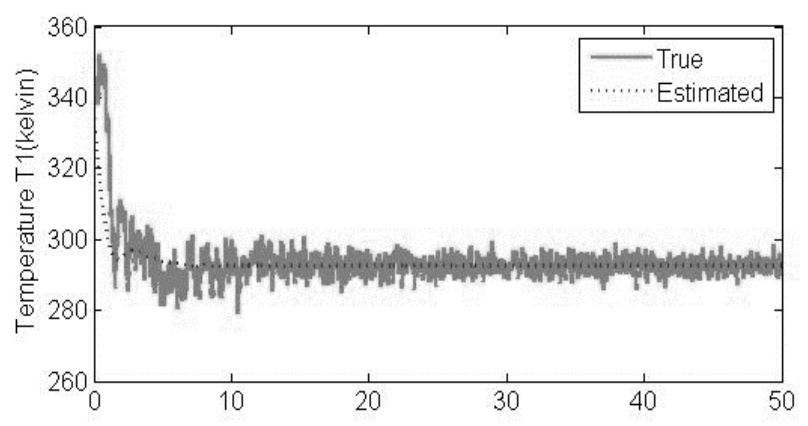

Time (min)

Fig. 11 Graph showing the plot of temperature of tank 1 against time (duration 50 min).

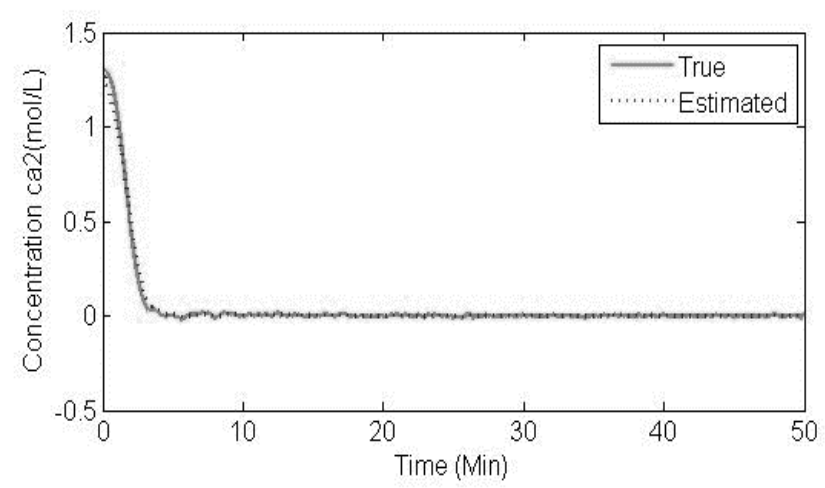

Fig. 12 Graph showing the plot of concentration of tank 2 against time (duration $50 \mathrm{~min}$ ).

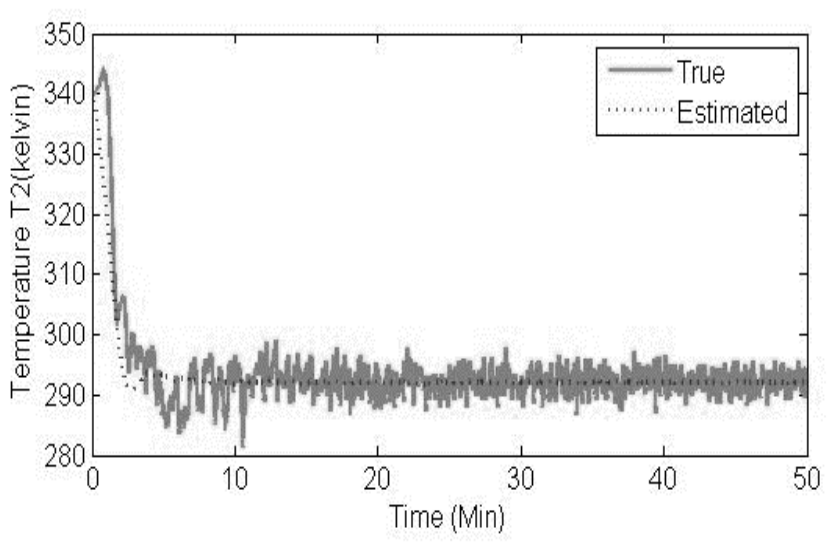

Fig. 13 Graph showing the plot of temperature of tank 2 against time (duration $\mathbf{5 0}$ min).

\section{With Fault}

When fault is introduced in the system, the output of the system looks like the following graphs:

\section{Heat Exchange coefficient Fault $\mathrm{C} \rho$ parameter fault}
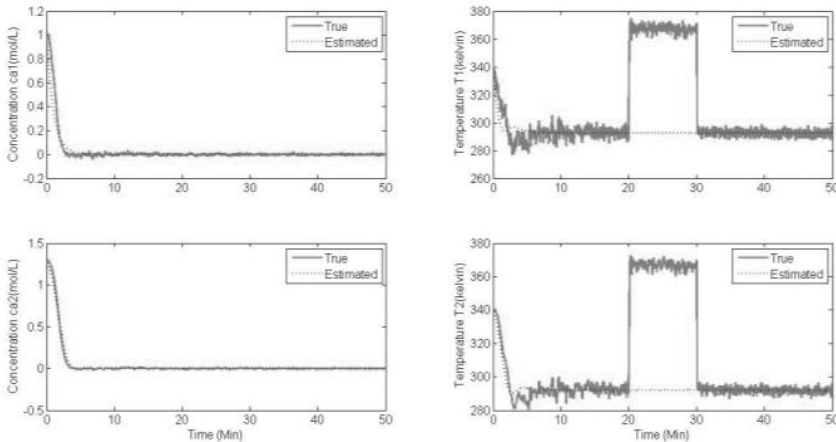

Time (min)

Fig. 14 Introduction of heat exchange coefficient fault in the dual tank CSTR system

In the above graph, heat exchange coefficient fault is introduced to the system. The duration of the fault is 10 minutes and is between 20 and 30 minutes. It can be inferred from the above graph that when the fault is introduced the temperatures of both the tanks are affected and the concentrations of the two tanks remain unaffected. 


\section{Reactant Feed Flow Fault}

\section{q parameter fault}
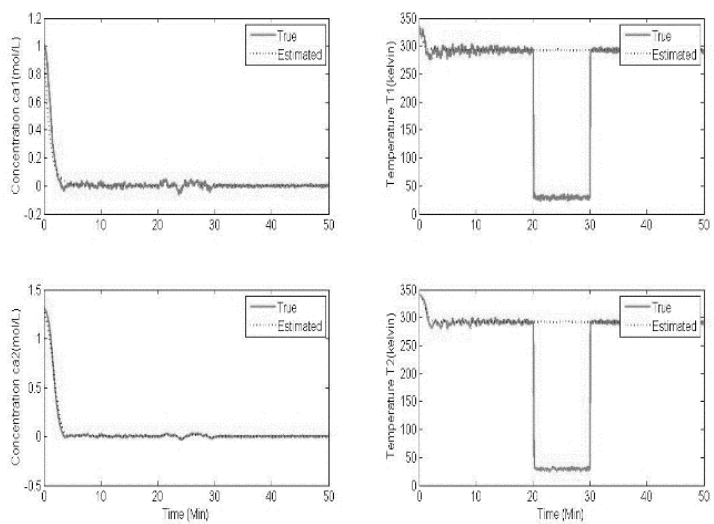

Time (min)

Fig. 15 Introduction of reactant feed flow fault in the dual tank CSTR system

In the above graph, reactant feed flow fault is introduced to the system. The duration of the fault is 10 minutes and is between 20 and 30 minutes. It can be inferred from the above graph that when the fault is introduced not only the temperatures of both the tanks are affected but also the concentrations of the two tanks are affected.

\section{Conclusion}

Kalman Filter works well for linear systems. In case of nonlinear systems, it should be linearized first and then Kalman Filter is implemented. So this is time consuming. Linearization process reduces the accuracy of the system. Extended Kalman Filter works on non-linear system and it linearizes at each estimate. From the results, Extended Kalman filter acts on non-linear system and tracks the system output more accurately when compared to Kalman filter. Even the fault of very small value is clearly visible in Extended Kalman filter.

\section{Future Scope}

The future scope is that this work can be extended to more accurate and reliable algorithms like the Unscented Kalman filter and the Particle Filter [5].The CSTR system can be replaced with any other non-linear or linear system.

\section{References}

- J. Getler, Fault Detection and Diagnosis in Engineering Systems, CRC Press, 1998.

- S. X.Ding, Model-Based Fault Diagnosis Techniques, Springer Publication, Second Edition, 2008.

- B. Khalali and J. Poshtan, "Fault detection of a CSTR using an Observer-based method and Particle
Filters," Journal of Control and Systems Engineering, vol. 1, no. 2, pp. 30-36, September, 2013.

- R. Isermann, Fault diagnosis systems, New York: Springer Berlin Heidelberg, 1997.

- D. Simon, Optimal State Estimation, John Wiley and Sons, 2006.

- J.Fichou, "Particle Based Methods for Parameter Estimation and Tracking: Numerical Experiments," Publication Interne IRISA, no. 1604, 2004.

- Michael A. Henson and Dale E. Seborg, "InputOutput Linearisation of General Nonlinear Processes," AICHE Journal, vol. 36, no. 11, pp. 1753-1758, November 1990.

- G. Stephanopolos, An Introduction to Theory and Practice, Pearson Education, 2012.

- Padmakumar S., Vivek Agarwal, "A Comparitive Study into Observer based Fault Detection and Diagnosis in DC Motors," World Academy of Science, vol. 3, 2009. 